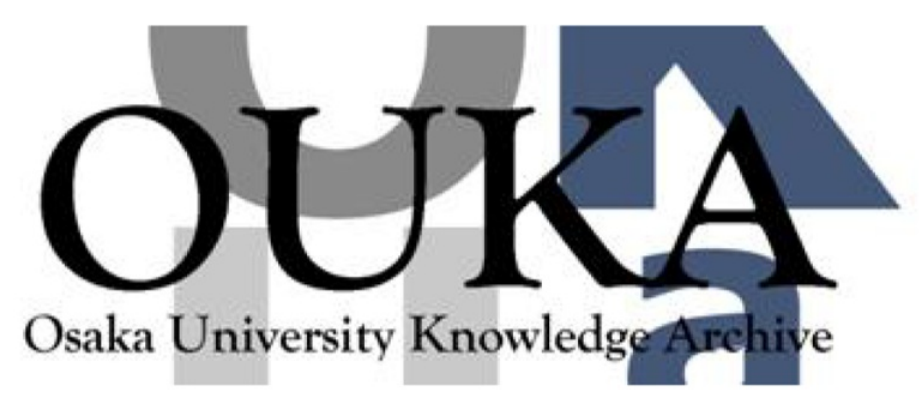

\begin{tabular}{|c|l|}
\hline Title & $\begin{array}{l}\text { Magnetic-field cycling instrumentation for } \\
\text { dynamic nuclear polarization-nuclear magnetic } \\
\text { resonance using photoexcited triplets }\end{array}$ \\
\hline Author(s) & $\begin{array}{l}\text { Kagawa, Akinori; Negoro, Makoto; Takeda, } \\
\text { Kazuyuki et al. }\end{array}$ \\
\hline Citation & $\begin{array}{l}\text { Review of Scientific Instruments. 80(4) } \\
\text { p. } 044705\end{array}$ \\
\hline Issue Date & $2009-04-28$ \\
\hline oaire:version & VoR \\
\hline URL & https://hdl. handle. net/11094/77651 \\
\hline rights & \\
\hline Note & \\
\hline
\end{tabular}

Osaka University Knowledge Archive : OUKA

https://ir. Library. osaka-u. ac. jp/

Osaka University 


\section{Magnetic-field cycling instrumentation for dynamic nuclear polarization-nuclear magnetic resonance using photoexcited triplets}

Cite as: Rev. Sci. Instrum. 80, 044705 (2009); https://doi.org/10.1063/1.3123346

Submitted: 28 March 2008 . Accepted: 31 March 2009 . Published Online: 28 April 2009

Akinori Kagawa, Makoto Negoro, Kazuyuki Takeda, and Masahiro Kitagawa

\section{ARTICLES YOU MAY BE INTERESTED IN}

Electron spin echoes of a photoexcited triplet: Pentacene in p-terphenyl crystals The Journal of Chemical Physics 75, 3746 (1981); https://doi.org/10.1063/1.442520

Zero-field magnetic resonance of the photo-excited triplet state of pentacene at room temperature

The Journal of Chemical Physics 113, 11194 (2000); https://doi.org/10.1063/1.1326069

${ }^{2} \mathrm{H}$-decoupling-accelerated ${ }^{1} \mathrm{H}$ spin diffusion in dynamic nuclear polarization with photoexcited triplet electrons

The Journal of Chemical Physics 133, 154504 (2010); https://doi.org/10.1063/1.3493453

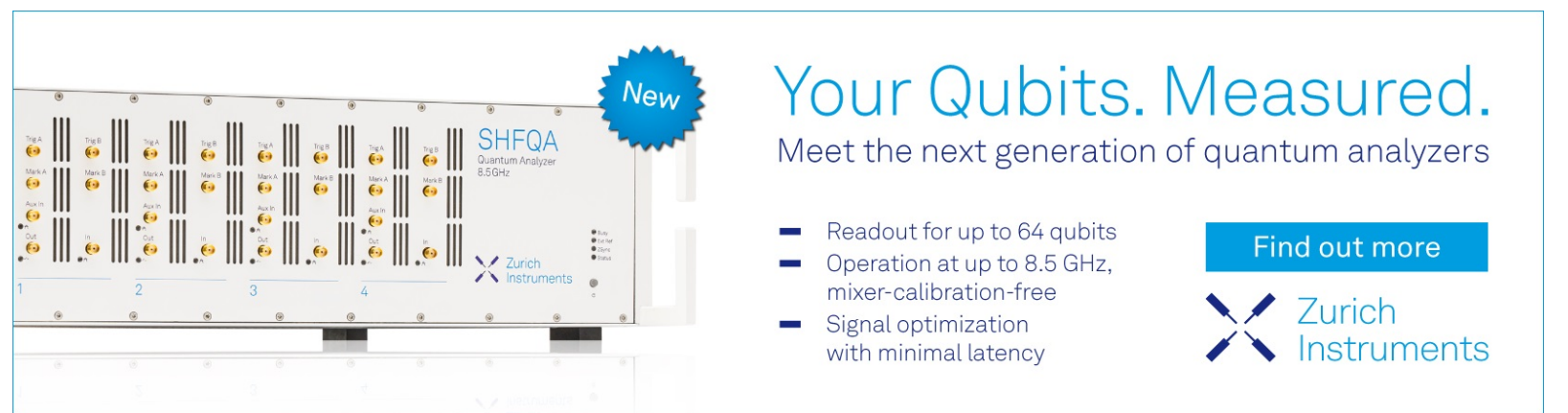




\title{
Magnetic-field cycling instrumentation for dynamic nuclear polarization-nuclear magnetic resonance using photoexcited triplets
}

\author{
Akinori Kagawa, ${ }^{1,2, a)}$ Makoto Negoro, ${ }^{1}$ Kazuyuki Takeda, ${ }^{3}$ and Masahiro Kitagawa ${ }^{1}$ \\ ${ }^{1}$ Graduate School of Engineering Science, Osaka University, 560-8531 Toyonaka, Japan \\ ${ }^{2}$ CREST, Japan Science and Technology Agency, Kawaguchi-shi, 332-0012 Saitama, Japan \\ ${ }^{3}$ Graduate School of Science, Kyoto University, 606-8502 Kyoto, Japan
}

(Received 28 March 2008; accepted 31 March 2009; published online 28 April 2009)

\begin{abstract}
To advance static solid-state NMR with hyperpolarized nuclear spins, a system has been developed enabling dynamic nuclear polarization (DNP) using electron spins in the photoexcited triplet state with $X$-band microwave apparatus, followed by static solid-state nuclear magnetic resonance (NMR) experiments using the polarized nuclear-spin system with a goniometer. In order to perform the DNP and NMR procedures in different magnetic fields, the DNP system and the NMR system are spatially separated, between which the sample can be shuttled while its orientation is controlled in a reproducible fashion. We demonstrate that the system developed in this work is operational for solid-state NMR with hyperpolarized nuclear-spin systems in static organic materials, and also discuss the application of our system. (C) 2009 American Institute of Physics.
\end{abstract}

[DOI: $10.1063 / 1.3123346$ ]

Sensitivity enhancement in nuclear magnetic resonance (NMR) spectroscopy has been of considerable interest, because it opens up possibilities for analyzing chemically/ biologically interesting, but so far, inaccessible materials. In order to "see the unseen," various techniques for enhancing nuclear-spin polarization and therefore the sensitivity by orders of magnitude have been applied by making use of the characteristics of the individual techniques. For example, hyperpolarized ${ }^{129} \mathrm{Xe}$ by optical pumping has been used for in vivo magnetic resonance imaging, ${ }^{1}$ porous materials, ${ }^{2}$ and surface science. ${ }^{3}$ In optical pumping of semiconductors, an attempt was made to enhance the nuclear-spin polarization in a thin film of biological material deposited on the surface of InP. $^{4}$ In optical nuclear polarization (ONP) ${ }^{5}$ molecular dynamics, phase transition ${ }^{6}$ and exciton dynamics ${ }^{7}$ were elucidated through measurements of the enhanced ${ }^{13} \mathrm{C}$ NMR signals. Also, the sensitivity of NMR spectra of biomolecules in frozen solutions was considerably enhanced by dynamic nuclear polarization $(\mathrm{DNP})^{8,9}$ using free radicals. Through technical improvements of high-frequency microwave power amplifiers, this method is widely applicable for liquid-state $\mathrm{NMR}^{10,11}$ and solid-state $\mathrm{NMR}^{12,13}$ because it can use high magnetic fields.

In contrast to DNP using free radicals, DNP using electron spins in the photoexcited triplet state, often called microwave-induced ONP (MIONP), ${ }^{1,15}$ is also an attractive strategy for significantly enhancing nuclear-spin polarization for the following reasons. (i) In some of the photoexcitable molecules, the transient electron spin polarization in the metastable photoexcited triplet state is much larger than that in free radicals in thermal equilibrium irrespective of temperature and magnetic field, eliminating the technically challenging demands for performing DNP both at cryogenic tem-

\footnotetext{
${ }^{\text {a)} E l e c t r o n i c ~ m a i l: ~ k a g a w a @ q c . e e . e s . o s a k a-u . a c . j p . ~}$
}

perature and in high magnetic fields requiring millimeterwave apparatus. (ii) Owing to the finite lifetime of the triplet electron spins, the nuclear-spin system becomes free from disturbance by the electron paramagnetism causing nuclear spin-lattice relaxation, decoherence, ${ }^{16}$ and line shape distortion simply by stopping photoexcitation once the electron-tonucleus polarization transfer has been complete. ${ }^{17}$ Using an efficient MIONP technique based on "cross polarization,"18 referred to as integrated solid effect (ISE), ${ }^{18-20}$ nuclear-spin polarization can be enhanced significantly. In the ISE pulse sequence depicted in Fig. 1, the magnetic-field sweep is applied together with microwave irradiation during the lifetime of the photoexcited triplet state so that the inhomogeneously broadened resonance line of the electron spin due to the hyperfine coupling with the surrounding protons is adiabatically swept over. By repeating the ISE sequence, proton polarization of 0.7 has been attained at $105 \mathrm{~K}$ and in $0.3 \mathrm{~T}$ in a single crystal of naphthalene doped with a small amount of pentacene as the triplet guest. ${ }^{21,22}$ Although such high attainable polarization is quite attractive and extensive fundamental studies on DNP using the electron spins in the photoexcited triplet state have been reported, its application to chemistry is not viable yet because of the low magnetic fields. To achieve the goal of utilizing the high polarization attainable by DNP using the triplet electron spins, we present an experimental setup that exploits the above attractive features of the electron spins in the photoexcited triplet state. DNP experiments by ISE are demonstrated using the electron spin in the photoexcited triplet state of pentacene, which is one of the most extensively investigated triplet materials. NMR experiments are demonstrated in single crystal samples in a magnetic field of $11.7 \mathrm{~T}$ with nuclear-spin species of ${ }^{1} \mathrm{H}$, ${ }^{13} \mathrm{C}$, and ${ }^{2} \mathrm{H}$. A goniometer is also developed to adjust alignment of the crystal samples in a reproducible manner. Finally, possible applications enabling such NMR experiments 


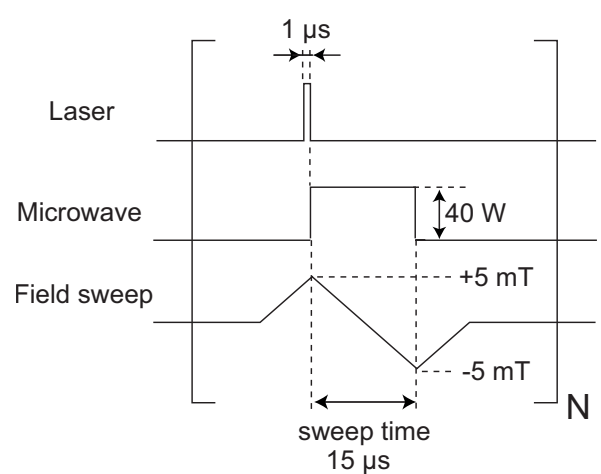

FIG. 1. A pulse sequence for ISE. After a laser pulse, microwave irradiation is applied together with a magnetic-field sweep so that the resonance line of the electron spin in the photoexcited triplet state is adiabatically swept over. The microwave intensity is adjusted to fulfill the Hartmann-Hahn condition between the electron spin in the rotating frame and the proton spin in the laboratory frame during the course of the adiabatic sweep. This sequence is repeated to build up the proton polarization.

that have so far been impossible, are discussed.

In DNP using free radicals, electron spins in thermal equilibrium are used. Since the equilibrium polarization increases with the magnetic field, recent DNP works are carried out using technically demanding millimeter-wave apparatus in high magnetic fields in which the NMR measurement is also useful. On the other hand, independence of the polarization of the electron spin in the photoexcited triplet state from the magnetic field lifts the restriction of carrying out DNP in the same high magnetic field as that for NMR measurements. That is, the DNP process can be performed in much lower magnetic fields without diminishing the available electron spin polarization. As a result, the microwave frequency for electron spin resonance is reduced and fabrication of the DNP apparatus becomes easier. Moreover, since the rf resonant circuit for NMR and microwave cavity for DNP are spatially well separated, both of them can be optimized without interference.

Separate experiments of DNP in the low field and NMR in the high field have been well established in a number of the previous works. On the other hand, what is challenging in this work is to carry out these experiments successively one after another by means of sample shuttling. Filed-cycling by sample shuttling has been reported in papers on zero-field NMR and nuclear quadruple resonance, ${ }^{23-26}$ nuclear-spin relaxation studies, ${ }^{27-29}$ coherence transfer in chemically induced dynamic nuclear polarization, ${ }^{30}$ and ONP. ${ }^{17,31-34}$

As schematically shown in Fig. 2, a split-electromagnet and a solenoidal superconducting magnet (SCM) are employed for DNP and NMR measurement, between which the sample is shuttled. The operation of sample shuttling is carried out in such a way that the orientation of the sample is controlled in both the DNP and the NMR parts with goniometers. The sample holder, to be docked and undocked with these goniometers, is described below. The sample is mechanically shuttled by pulling a nylon string within ca. $5 \mathrm{~s}$. Thus, our field-cycling system is suitable for such target nuclei that have spin-lattice relaxation times much longer than on the order of second. In order to maximize the eventual nuclear polarization, nuclear spin-lattice relaxation time is desirable to be as long as possible. Thus, the sample is thermally insulated with a double-layered glass tube, and can be
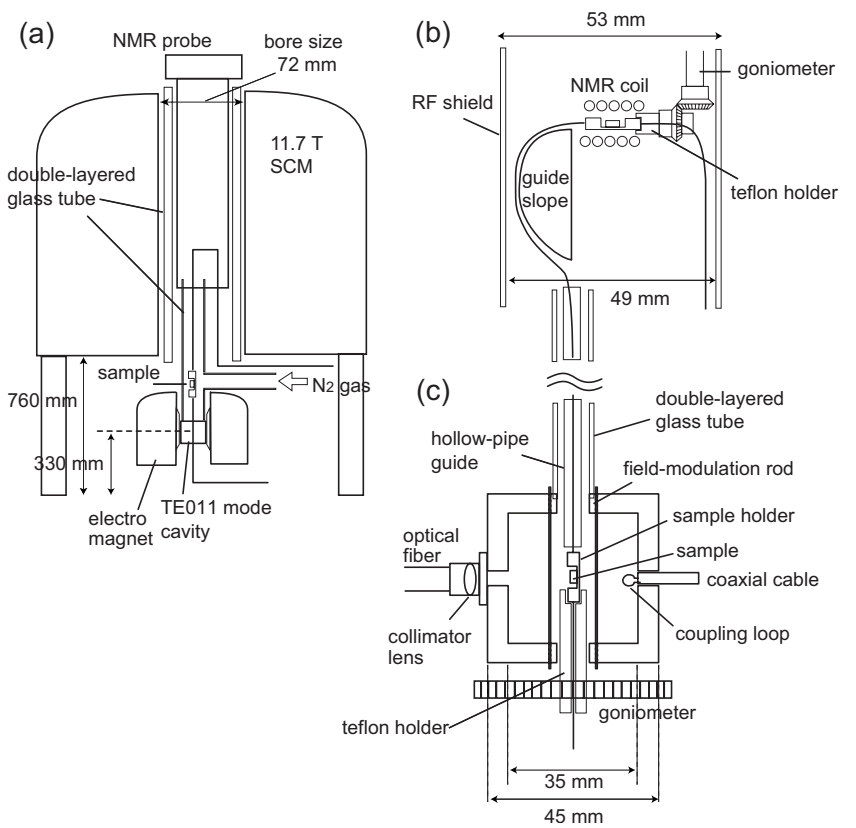

FIG. 2. (a) Schematic diagram of a field-cycling triplet-DNP system. The sample is to be shuttled between the high magnetic field (11.7 T) in a SCM and the low field $(\sim 0.3 \mathrm{~T})$ in an electromagnet. The system is thermally insulated in a vacuum double-layered glass tube, and the sample can be cooled with nitrogen gas flow. In the high field, the sample moves along a guide slope toward inside the NMR coil until the sample holder is docked with the goniometer, as described in (b). The NMR coil is triply tuned at 500, 125, and $76 \mathrm{MHz}$ for ${ }^{1} \mathrm{H},{ }^{13} \mathrm{C}$, and ${ }^{2} \mathrm{H}$ NMR experiments in $11.7 \mathrm{~T}$. In the low field, a TE011 mode $X$-band cavity is employed as described in (c), and a laser beam and $X$-band microwave irradiations can be applied together with magnetic-field sweep by means of a quadruple coil which is composed of four field-modulation rods. The sample holder can also be docked with the goniometer assembled with the cavity.

cooled with nitrogen gas flow down to $77 \mathrm{~K}$ so as to suppress spin-lattice relaxation, but not to enhance the thermal polarization. Also, thermal insulation is necessary not only in the DNP part of the system but also in the NMR part to prevent the sample from cracking due to rapid temperature change.

We intend to carry out the ISE sequence in a low magnetic field so that polarization transfer efficiency does not degrade, because the lower the magnetic field for DNP and microwave frequency for electron spin resonance, the less difficult it is to fabricate the microwave circuitry. Moreover, since the process of DNP by ISE relies on the HartmannHahn condition between the electron spin in the rotating frame and the nuclear spin in the laboratory frame, the less powerful amplifier suffices for microwave irradiation as the magnetic field is decreased. The lowest possible field is limited by the adiabatic condition for the field sweep over the resonance line of the electron spin packets. In the case of ISE, the adiabatic condition becomes stricter as the magnetic field is decreased, because the nuclear Zeeman interaction in the laboratory frame is scaled down and the less intense microwave irradiation should be used to satisfy the HartmannHahn condition. In order to satisfy the adiabatic condition, the magnetic-field sweep has to be performed slowly enough. However, the field sweep over the inhomogeneously broadened resonance line of the electron spin packets up to $\sim 10 \mathrm{mT}$ should be completed within the lifetime of the triplet state, which is on the order of tens of microseconds. From this consideration, we have chosen to use a magnetic field of 
around $0.3 \mathrm{~T}$, in which the microwave frequency for electron spin resonance is in the $X$-band region.

For the ISE experiment, we have developed a TE011 mode cylindrical $X$-band microwave cavity equipped with an optical window and a field-modulation coil, as shown in Fig. 2 (c). Its resonance frequency and the unloaded $Q$-factor were found to be $9.065 \mathrm{GHz}$ and 1200 , respectively.

As shown in Fig. 1, the ISE sequence requires, in addition to laser irradiation for photoexcitation and microwave irradiation at the electron spins in the photoexcited triplet state, an adiabatic magnetic-field sweep over the resonance line of the triplet electron spins. Also, when a single crystal sample is used, it is critical to optimize the crystal alignment with respect to the external magnetic field so as to maximize the orientation-dependent electron spin polarization in the photoexcited triplet state and thereby the attainable nuclearspin polarization in the DNP experiment. Although a twoaxis goniometer is needed for arbitrary crystal alignment, a mechanically simpler uniaxial goniometer suffices for setting the optimal crystal alignment when the crystal is appropriately mounted on the sample holder.

For the fast buildup of proton polarization, studies on buildup behavior of proton polarization by ISE in partially deuterated naphthalene are also in progress. For NMR experiments in such partially deuterated organic materials, we require that the NMR sample coil is triply tuned at the resonance frequencies of ${ }^{1} \mathrm{H},{ }^{13} \mathrm{C}$, and ${ }^{2} \mathrm{H}$ spins. As demonstrated below, the ${ }^{2} \mathrm{H}$ channel can serve for improving spectral resolution through by ${ }^{2} \mathrm{H}$-double-quantum decoupling. Also, another goniometer is necessary for manipulating the angle between the magnetic field and the target material in the NMR part of the system. For NMR experiments in the high field, a $11.7 \mathrm{~T}$ SCM was used together with a home-built NMR spectrometer ${ }^{35,36}$ and a home-built ${ }^{1} \mathrm{H}-{ }^{13} \mathrm{C}-{ }^{2} \mathrm{H}$ tripleresonance probe based on the design proposed by Martin et $a .^{37}$ and tuned at 500, 125 , and $76 \mathrm{MHz}$, respectively.

A single crystal sample of ca. $0.01 \mathrm{~mol} \%$ pentacenedoped naphthalene was grown by the Bridgman method. The sample size cut along the cleavage plane ( $a b$-plane) was $\sim 1.2 \times 1.4 \times 1.6 \mathrm{~mm}^{3}$. In order to maximize the electron spin polarization in the photoexcited triplet state of pentacene, the crystal was aligned in such a way that the long axis of the pentacene molecule is parallel to the external field. ${ }^{21,38}$ For photoexcitation of pentacene, a flashlamp-pumped dye laser (Cynosure, LFDL-3) with a pulse width of $1 \mu$ s and wavelength of $590 \mathrm{~nm}$ was used. The laser beam was fed through an optical fiber and led to the sample at which the beam diameter was adjusted to ca. $3 \mathrm{~mm}$ with a collimator lens. The power of the laser beam was $8 \mathrm{~mJ} / \mathrm{pulse}$, which is sufficient to photoexcite all pentacene molecules in the sample. ${ }^{39}$ The magnetic field in the DNP system was set to $0.2958 \mathrm{~T}$, corresponding to the transition between the $|-1\rangle$ and $|0\rangle$ sublevels in the photoexcited triplet state of the pentacene molecule when its long axis is parallel to the magnetic field.

${ }^{1} \mathrm{H}$ polarization was built up by repeating the ISE sequence shown in Fig. 1 at a rate of $50 \mathrm{~Hz}$ in the low-field DNP system. After the buildup process of ${ }^{1} \mathrm{H}$ polarization, the sample was shuttled into the high-field NMR system through a rectangular hollow-pipe guide placed inside the double-layered glass tube [Fig. 3(b) and 3(c)]. The hollow- (a) scale $[\mathrm{mm}]$

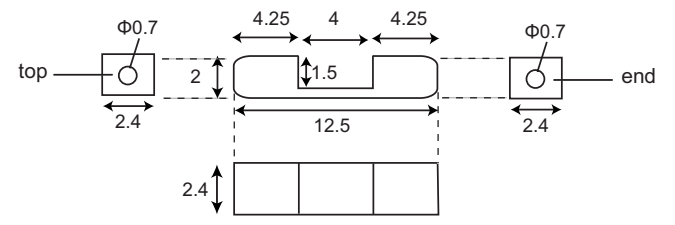

(b)

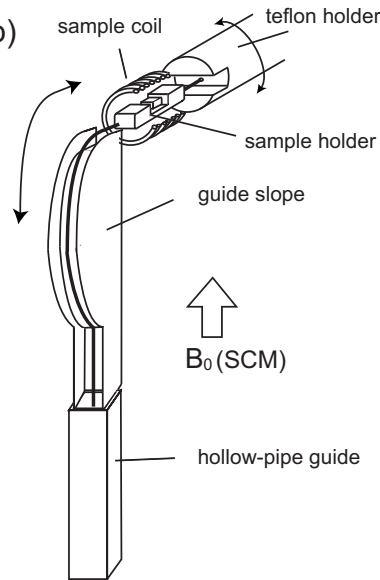

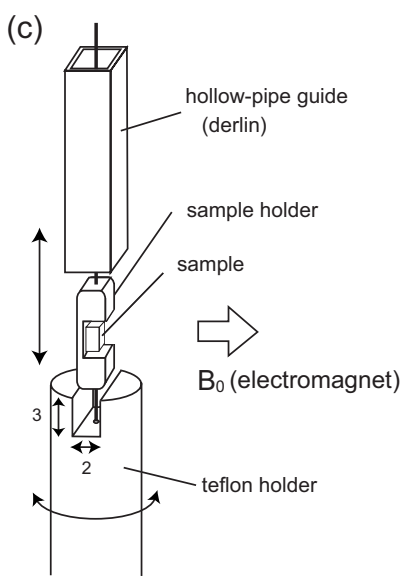

(c)
FIG. 3. (a) A drawing of a sample holder. The size is slightly smaller than diameter of solenoid coil in the NMR probe. Nylon strings $(\phi 0.45 \mathrm{~mm})$ are tied to both the top and end surface holes $(\phi 0.7 \mathrm{~mm}$, depth $3 \mathrm{~mm})$. (b) A schematic diagram of sample shuttling in the NMR probe. The sample holder slides along a guide slope toward inside the solenoid NMR coil until the sample holder is docked with a ditched Teflon holder on the goniometer. (c) A schematic diagram of sample shuttling in the $X$-band cavity. The ditched Teflon holder is also used. A hollow-pipe guide, which has rectangular cross section $\left(3 \times 2.3 \mathrm{~mm}^{2}\right)$ slightly larger than the size of sample holder $\left(2.4 \times 2 \mathrm{~mm}^{2}\right)$, prevents a turn of the sample holder.

pipe guide, which has a cross section $\left(3 \times 2.3 \mathrm{~mm}^{2}\right)$ slightly larger than the size of the top-end of the sample holder [Fig. 3(a)], prevents the sample holder from turning. As described in Fig. 3(b), the sample is then slid along a guide slope toward inside the solenoid NMR coil until the sample holder was docked with a ditch on the goniometer assembled in the NMR probe. The same mechanism is also employed for docking the sample holder with the DNP system [Fig. 3(c)]. The goniometer axis normal to the magnetic field inside the SCM demands the sample to be shuttled along a curved path. At the cost of this mechanical complexity, however, a solenoid coil can be used so that the efficiency of rf irradiation and the detection sensitivity will become higher than in the previous shuttling systems along a straight path where a saddle coil is the natural choice. The goniometer has a $\pm 180^{\circ}$ range of movement with an accuracy $1.8^{\circ}$. The attained rf intensities $\left(\gamma B_{1} / 2 \pi, \gamma\right.$ is the gyromagnetic ratio) with $300 \mathrm{~W}$ power amplifiers were 130,110 , and $55 \mathrm{kHz}$ for the ${ }^{1} \mathrm{H},{ }^{13} \mathrm{C}$, and ${ }^{2} \mathrm{H}$ rf channels, respectively.

In order to examine the performance of the system we have developed in this work, the following three experiments were carried out. The first one is the buildup test of ${ }^{1} \mathrm{H}$ polarization by repeating the ISE sequence for $90 \mathrm{~min}$ at a rate of $50 \mathrm{~Hz}$. Figure 4(a) shows a ${ }^{1} \mathrm{H}$ magic sandwich echo ${ }^{40,41}$ spectrum measured in the high field. By comparing the ${ }^{1} \mathrm{H}$ NMR signal intensity with that obtained in the liquid-state ethanol in thermal equilibrium, the enhanced ${ }^{1} \mathrm{H}$ polarization 
(a)

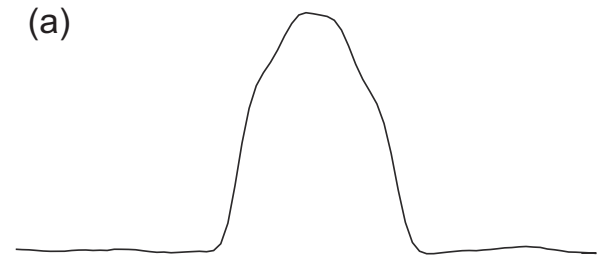

(b)

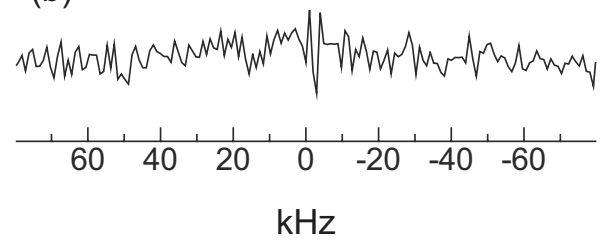

FIG. 4. Single-scan ${ }^{1} \mathrm{H}$ NMR spectra in a single crystal sample of pentacene-doped naphthalene in $11.7 \mathrm{~T}$ and obtained (a) after repeating the ISE sequences at a rate of $50 \mathrm{~Hz}$ for $90 \mathrm{~min}$ at $203 \mathrm{~K}$, and (b) without the ISE sequence in thermal equilibrium at room temperature $(\times 50)$. These experiments were performed without a preamplifier to prevent the acquisition system from saturating.

was estimated to be 0.11 , which corresponds to $\sim 74000$ (1900) times that in thermal equilibrium in $0.2958 \mathrm{~T}(11.7 \mathrm{~T})$ at $203 \mathrm{~K}$. According to the temperature dependence of spinlattice relaxation time of proton in a single crystal of naphthalene in a field of $94 \mathrm{mT}^{42}$ the proton spin in naphthalene has a sufficient long spin-lattice relaxation time for the ISE experiment at $203 \mathrm{~K}^{43}$ The enhanced ${ }^{1} \mathrm{H}$ signal was so strong that the gain in the NMR receiver was significantly reduced to prevent the acquisition system from saturating. The loss of the ${ }^{1} \mathrm{H}$ polarization due to sample shuttling was found to be negligible, since we found no discernible reduction in the signal intensity even when the sample was shuttled between the high and low fields several times before NMR measurement.

The second NMR experiment, demonstrated in Fig. 5, is ${ }^{1} \mathrm{H}-{ }^{13} \mathrm{C}$ cross polarization, ${ }^{44,45}$ by which the enhanced polarization of the ${ }^{1} \mathrm{H}$ spins in naphthalene can further be transferred to the $\sim 1 \%$ naturally abundant ${ }^{13} \mathrm{C}$ spins. In a single crystal sample, the resonance frequency of the individual ${ }^{13} \mathrm{C}$ spin depends on the principal values and the orientation of the chemical shift tensor with respect to the external magnetic field. Thus, the profile of the ${ }^{13} \mathrm{C}$ NMR spectra varied as the goniometer was rotated, as demonstrated in Fig. 5. Each ${ }^{13} \mathrm{C}$ spectrum in Fig. 5 was obtained after building up the ${ }^{1} \mathrm{H}$ polarization by DNP in the low field. Even when the sample holder was repeatedly docked and undocked with the goniometer, the orientational reproducibility was good, as demonstrated in the spectrum Fig. 5(e) obtained after measuring the spectrum in Fig. 5(d) and restoring the goniometer to the original orientation in which the spectrum in Fig. 5(a) was measured. The experimental results showed that our triple-resonance NMR probe can be applied to static oriented samples.

The third experiment was carried out using pentacenedoped $99.4 \%$ deuterated naphthalene for examining the performance of the ${ }^{2} \mathrm{H}$ channel, and the polarization of the residual ${ }^{1} \mathrm{H}$ spins in this heavily deuterated sample was built up by ISE. As shown in Fig. 6, the resonance linewidth of the ${ }^{1} \mathrm{H}$ spectrum was found to be considerably smaller than that obtained in the undeuterated sample shown in Fig. 4 due to reduction in the ${ }^{1} \mathrm{H}-{ }^{1} \mathrm{H}$ dipolar interactions by dilution.

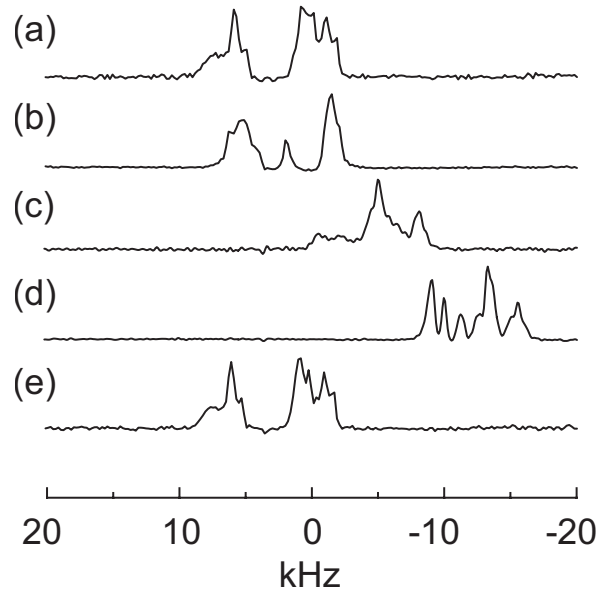

FIG. 5. Single-scan natural-abundance ${ }^{13} \mathrm{C}$ NMR spectra in a single crystal sample of pentacene-doped naphthalene. After the buildup process of the ${ }^{1} \mathrm{H}$ polarization by, repeating the ISE sequence at a repetition rate of $50 \mathrm{~Hz}$ for $10 \mathrm{~min}$, the sample was shuttled into the NMR probe in the high field, where ${ }^{1} \mathrm{H}-{ }^{13} \mathrm{C}$ cross polarization was performed before measuring the ${ }^{13} \mathrm{C}$ NMR signal under ${ }^{1} \mathrm{H}$ decoupling. Spectra in (a)-(d) were obtained with different crystal alignments by setting the goniometer angle in advance to the sample shuttling. The relative goniometer angle in (b)-(d) with respect to that in (a) were $30^{\circ}, 60^{\circ}$, and $90^{\circ}$. In order to verify the reproducibility of the crystal orientation, the spectrum shown in (e) was measured after obtaining the spectra in (a)-(d) by restoring the goniometer angle to that used for (a). Since the efficiency of cross polarization from the ${ }^{1} \mathrm{H}$ spins to the ${ }^{13} \mathrm{C}$ spins depends on the orientation of the internuclear vector, the signal-to-noise ratios are different from one another.

Since the individual ${ }^{1} \mathrm{H}$ spin is now surrounded by a number of ${ }^{2} \mathrm{H}$ spins, the resonance linewidth is mainly determined by the dipolar interactions between the ${ }^{1} \mathrm{H}$ and ${ }^{2} \mathrm{H}$ spins, which can be eliminated by double-quantum ${ }^{2} \mathrm{H}$ decoupling, ${ }^{46}$ as demonstrated in Fig. 4.

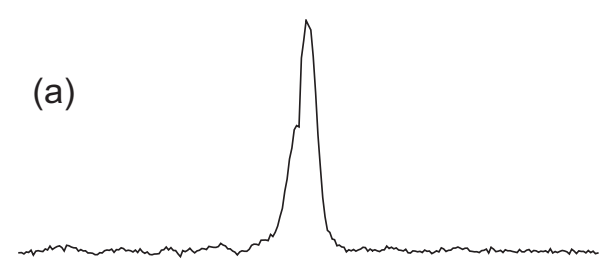

(b)

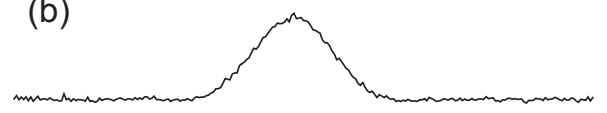

(c)

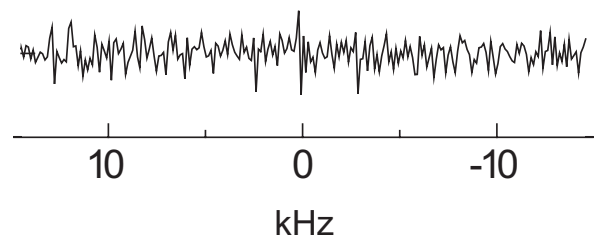

FIG. 6. Single-scan ${ }^{1} \mathrm{H}$ NMR spectra in a single crystal sample of pentacene-doped $99.4 \%$-deuterated naphthalene. By repeating the ISE sequence at a rate of $50 \mathrm{~Hz}$, the polarization of the residual proton spins were built up for $10 \mathrm{~min}$. The spectra in (a) and (b) were obtained with and without ${ }^{2} \mathrm{H}$ double-quantum decoupling. For comparison, the spectrum without ${ }^{1} \mathrm{H}$ polarization buildup by ISE is shown in $(\mathrm{c})(\times 10)$. 
As shown in the above three experimental demonstrations, the field-cycling system described here opens up the possibility of NMR measurements of what has been unobservable so far. For the application of the filed-cycling system developed in this work, we considered the following strategies. First, we plan to use pentacene/naphthalene or pentacen/p-terphenyl system as a source of high polarization, aiming at transferring polarization to the target molecules through ${ }^{1} \mathrm{H}$ spin diffusion. $P$-terphenyl is another candidate for a host in ISE experiments, since the polarization was higher than that of naphthalene at room temperature due to high concentrations of pentacene. ${ }^{47}$ The first approach is the application to observation of codoping other target molecules in the single crystals of the source or microscopically mixing the source with target molecules by solution freezing such as used in DNP using free radicals. ${ }^{12}$ The buildup process of DNP takes place only inside the source. Therefore, the finally attainable polarization is governed by the spin-lattice relaxation time of the source. In this case, we do not take into account the spin-lattice relaxation time of the target molecules, because the target molecules are easily polarized by spin diffusion of neighboring ${ }^{1} \mathrm{H}$ spins in the source. Since the spin-lattice relaxation time of naphthalene or $p$-terphenyl is on the order of $10 \mathrm{~min}$ even at room temperature, ${ }^{47}$ the reduction in the polarized magnetization is negligible during sample shuttling. Second, we have strategies for transferring the high polarization to the outside of the source. Recently, amyloidogenic peptide nanocrystals were polarized in a glycerol-water containing a biradical polarizing agent by thermal mixing method ${ }^{48}$ Also, we polarized polycrystalline samples of pentacene/naphthalene, ${ }^{22,49}$ which were pressed into a fine powder. These results implied that polycrystalline mixtures of the source and target molecules can be polarized by ISE sequences. Another possibility of the application to biochemistry, such as transferred optically pumped NMR (TOPNMR), ${ }^{4}$ is to enhance an aligned overlayer (e.g., membrane), which is deposited or adsorbed on the crystal surface of the source by spin coating, Langmuir-Blodgett method, or vapor deposition. Owing to a small diffusion barrier, ${ }^{48}$ the efficiency of transferring polarization from the source to the target molecules is higher than that of TOPNMR, which was assumed to be $\sim 5 \%$. ${ }^{4}$ A typical ${ }^{1} \mathrm{H}$ spin diffusion rate in ${ }^{1} \mathrm{H}$-rich organic solids is on the order of $10^{-16} \mathrm{~m}^{2} / \mathrm{s} .{ }^{50}$ Thus, the appropriate sizes (e.g., the diameter of the particle or the thickness of the overlayer) are $<1 \mu \mathrm{m}$ in order to enhance the whole region of the target molecules. In these cases, the target molecules should have long spin-lattice relaxation time as compared to the buildup process and the shuttling time. In the future, we will examine other triplet-molecules ${ }^{51}$ or host molecules using a laser, which has a suitable wavelength for each material. For instance, tryptophan, tyrosine, and phenylalanine are photoexcitable to the triplet state. ${ }^{52-54}$ Those materials may be applicable to enhance the sensitivity in peptides and proteins. Finally, DNP used in the electron spins in the photoexcited triplet state is also promising when the high polarization, not necessarily signal intensity, is essential. The initialization problem of an NMR quantum computer $^{55-59}$ is an example of such, where its true demonstration demands nuclear polarization beyond the entanglement threshold of the order 0.1, which depends on the number of qubits involved in the spin system..$^{60,61}$
Although DNP using triplet electron spins by ISE has the favorable properties described above, the buildup rate is slow relative to DNP using free radicals by thermal mixing. ${ }^{12}$ In order to both attain the higher nuclear polarization and in shorter experimentation time, we have the following two strategies. First, we plan to construct a laser with a high repetition rate than $50 \mathrm{~Hz}$ with appropriate beam power, pulse width, and a wavelength. The current maximum ISE repetition rate of $50 \mathrm{~Hz}$ is much lower than the limit given by the inverse of the lifetime of the triplet state, which is, when the lifetime is $100 \mu$ s for instance, $10 \mathrm{kHz}$. The higher the repetition rate, the faster the buildup rate would be until the capacity of polarization transportation by spin diffusion saturates without considering thermal deterioration. Second, studies on optimization of ${ }^{1} \mathrm{H}$ spin density by partial deuteration of the host crystal are in progress. ${ }^{62}$ The partial deuteration method is also used for DNP using free radicals. ${ }^{63}$ By the improvements, the attainable polarization is expected to be $\sim 10 \%$ even at room temperature for several minutes. Therefore our instrumentation has the possibility of application to NMR experiments at room temperature.

To summarize, we have developed a field-cycling system for ISE experiment and ${ }^{1} \mathrm{H}-{ }^{13} \mathrm{C}-{ }^{2} \mathrm{H}$ triple resonance NMR, which serves for static solid-state NMR with high polarization. At the cost of mechanical complexity using the solenoid NMR coil where the sample is shuttled along the curved path, we could employ the goniometer perpendicular to the magnetic field, which is convenient for studying oriented materials such as liquid crystals and lipid bilayers. Furthermore, the efficiency of rf irradiation and the detection sensitivity has become higher than that in the previous shuttling system along a straight path where a saddle coil is a natural choice. The experiments demonstrated in this paper confirm that the hardware developed can be used for structure analysis by static solid-state NMR with high polarization. When the magnetic field for NMR measurement is altered, hardware modification is only needed to the NMR tuning circuit, since the DNP and NMR apparatuses are spatially separated. This is in contrast to DNP using free radicals, where the magnetic field for DNP should also be high so as to use as large equilibrium polarization as possible, which would require reconstruction of somewhat technically challenging and costly millimeter-wave equipment.

This work has been supported by the CREST program of Japan Science and Technology Agency. A.K. and M.N. would like to thank 21st Century Center of Excellence $(\mathrm{COE})$ program for financial support.

${ }^{1}$ T. G. Walker and W. Happer, Rev. Mod. Phys. 69, 629 (1997).

${ }^{2}$ M. A. Springuel-Huet and J. Fraissard, Chem. Phys. Lett. 154, 299 (1989).

${ }^{3}$ D. Raftery, H. Long, T. Meersmann, P. J. Grandinetti, L. Reven, and A. Pines, Phys. Rev. Lett. 66, 584 (1991).

${ }^{4}$ R. Tycko, Solid State Nucl. Magn. Reson. 11, 1 (1998).

${ }^{5}$ G. Maier, U. Haeberlen, H. C. Wolf, and K. H. Hausser, Phys. Lett. 25, 384 (1967).

${ }^{6}$ J. A. Ripmeester and A. H. Reddoch, J. Chem. Phys. 74, 1526 (1981).

${ }^{7}$ J. M. Park and A. H. Reddoch, J. Chem. Phys. 74, 1519 (1981).

${ }^{8}$ A. Abragam, Principles of Nuclear Magnetism (Clarendon, Oxford, 1961).

${ }^{9}$ A. Abragam and M. Goldman, Nuclear Magnetism: Order and Disorder (Clarendon, Oxford, 1982).

${ }^{10}$ J. H. Ardenkjær-Larsen, B. Fridlund, A. Gram, G. Hansson, L. Hansson, M. H. Lerche, R. Servin, M. Thaning, and K. Golman, Proc. Natl. Acad. 
Sci. U.S.A. 100, 10158 (2003).

${ }^{11}$ J. Wolber, F. Ellner, B. Fridlund, A. Gram, H. Jóhannesson, G. Hansson, L. H. Hasson, M. H. Lerche, S. Månsson, R. Servin, M. Thaning, K. Golman, and J. H. Ardenkjær-Larsen, Nucl. Instrum. Methods Phys. Res. A 526, 173 (2004).

${ }^{12}$ D. A. Hall, D. C. Maus, G. J. Gerfen, S. J. Inati, L. R. Becerra, F. W. Dahlquist, and R. G. Griffin, Science 276, 930 (1997).

${ }^{13}$ T. Maly, G. T. Debelouchina, V. S. Bajaj, K.-N. Hu, C.-G. Joo, M. L. Mark-Jurkauskas, J. R. Sirigiri, P. C. A. van der Wel, J. Herzfeld, R. J. Temkin, and R. G. Griffin, J. Chem. Phys. 128, 052211 (2008).

${ }^{14}$ M. Deimling, H. Brunner, K. P. Dinse, and K. H. Hausser, J. Magn. Reson. (1969-1992) 39, 185 (1980).

${ }^{15}$ H. W. van Kesteren, W. Th. Wenckebach, and J. Schmidt, Phys. Rev. Lett. 55, $1642(1985)$

${ }^{16}$ N. Bloembergen, Physica 15, 386 (1949).

${ }^{17}$ D. Stehlik and H. M. Vieth, Pulsed Magnetic Resonance NMR, ESR and Optics (Oxford University Press, New York, 1992), p. 446.

${ }^{18}$ A. Henstra, T.-S. Lin, J. Schmidt, and W. Th. Wenckebach, Chem. Phys. Lett. 165, 6 (1990).

${ }^{19}$ M. Inuma, I. Shaké, R. Takizawa, M. Daigo, H. M. Shimizu, Y. Takahashi, A. Masaike, and T. Yabuzaki, Phys. Lett. A 208, 251 (1995).

${ }^{20}$ M. Iinuma, Y. Takahashi, I. Shaké, M. Oda, A. Masaike, T. Yabuzaki, and H. M. Shimizu, Phys. Rev. Lett. 84, 171 (2000).

${ }^{21}$ K. Takeda, K. Takegoshi, and T. Terao, J. Phys. Soc. Jpn. 73, 2313 (2004).

${ }^{22}$ K. Takeda, K. Takegoshi, and T. Terao, J. Phys. Soc. Jpn. 73, 2319 (2004).

${ }^{23}$ D. P. Weitekamp, A. Bielecki, D. Zax, K. Zilm, and A. Pines, Phys. Rev. Lett. 50, 1807 (1983).

${ }^{24}$ A. M. Thayer, J. M. Millar, and A. Pines, Chem. Phys. Lett. 129, 55 (1986).

${ }^{25}$ A. Bielecki, D. B. Zax, K. W. Zilm, and A. Pines, Rev. Sci. Instrum. 57, 393 (1986)

${ }^{26}$ R. Kreis, A. Thomas, W. Studer, and R. R. Ernst, J. Chem. Phys. 89, 6623 (1988).

${ }^{27}$ A. G. Redfield, Magn. Reson. Chem. 41, 753 (2003).

${ }^{28}$ K. Victor, V. Kavolius, and R. G. Bryant, J. Magn. Reson. 171, 253 (2004).

${ }^{29}$ H. Stork, M. Ditter, H. Pößer, A. F. Privalov, and F. Fujara, J. Magn. Reson. 192, 173 (2008)

${ }^{30}$ K. Miesel, K. L. Ivanov, A. V. Yurkovskaya, and H.-M. Vieth, Chem. Phys. Lett. 425, 71 (2006).

${ }^{31}$ R. Tycko, A. Pines, and D. Stehlik, Chem. Phys. Lett. 93, 392 (1982).

${ }^{32}$ M. Benkert and H.-M. Vieth, Chem. Phys. Lett. 230, 153 (1994).

${ }^{33}$ J. Allgeier, G. Buntkowsky, S. Hentrich, M. Nack, and H.-M. Vieth, Ber. Bunsenges. Phys. Chem 93, 1281 (1989).

${ }^{34}$ G. Buntkowsky, W. Hoffmann, T. Kupka, G. Pasterna, M. Jaworska, and H.-M. Vieth, J. Phys. Chem. A 102, 5794 (1998).

${ }^{35}$ K. Takeda, Rev. Sci. Instrum. 78, 033103 (2007).

${ }^{36}$ K. Takeda, J. Magn. Reson. 192, 218 (2008).

${ }^{37}$ R. W. Martin, E. K. Paulson, and K. W. Zilm, Rev. Sci. Instrum. 74, 3045 (2003).
${ }^{38}$ D. J. Sloop, T.-L. Yu, T.-S. Lin, and S. I. Weissman, J. Chem. Phys. 75, 3746 (1981).

${ }^{39}$ K. Takeda, K. Takegoshi, and T. Terao, J. Chem. Phys. 117, 4940 (2002).

${ }^{40}$ W.-K. Rhim, A. Pines, and J. S. Waugh, Phys. Rev. B 3, 684 (1971).

${ }^{41}$ C. P. Slichter, Principles of Magnetic Resonance, Third Enlarged and Updated Edition (Springer, New York, 1990).

${ }^{42}$ J. U. von Schütz and H. C. Wolf, Z. Naturforsch. A 27, 42 (1972).

${ }^{43}$ M. Iinuma, Y. Takahashi, I. Shaké, M. Oda, H. M. Shimizu, A. Masaike, and Y. Yabuzaki, J. Phys. Soc. Jpn. 74, 2622 (2005).

${ }^{44}$ A. Pines, M. G. Gibby, and J. S. Waugh, J. Chem. Phys. 59, 569 (1973).

${ }^{45} \mathrm{M}$. Mehring, Principles of high resolution NMR in solids (Springer, New York, 1983).

${ }^{46}$ A. Pines, D. J. Ruben, S. Vega, and M. Mehring, Phys. Rev. Lett. 36, 110 (1976).

${ }^{47}$ M. Iinuma, Y. Takahashi, I. Shakeé, M. Oda, A. Masaike, T. Yabuzaki, and H. M. Shimizu, Nucl. Instrum. Methods Phys. Res. A 529, 199 (2004).

${ }^{48}$ P. C. A. van der Wel, K.-N. Hu, J. Lewandowski, and R. G. Griffin, J. Am. Chem. Soc. 128, 10840 (2006).

${ }^{49}$ K. Takeda, K. Takegoshi, and T. Terao, Chem. Phys. Lett. 345, 166 (2001).

${ }^{50} \mathrm{~K}$. Schmidt-Rohr and H. W. Spiess, Multidimensional Solid-State NMR and Polymers, (Academic, London, 1994).

${ }^{51}$ R. S. Becker, Theory and Interpretation of Fluorescence and Phosphorescence (Wiley, New York, 1969).

${ }^{52}$ A. Ozarowski, A. Misra, S. Ghosh, and A. H. Maki, J. Phys. Chem. B 106, 5099 (2002).

${ }^{53}$ A. Ozarowski, J. Q. Wu, and A. H. Maki, Chem. Phys. Lett. 286, 433 (1998).

${ }^{54}$ J. Zuclich, J. Chem. Phys. 52, 3586 (1970).

${ }^{55}$ N. A. Gershenfeld and I. L. Chuang, Science 275, 350 (1997).

${ }^{56}$ D. G. Cory, R. Laflamme, E. Knill, L. Viola, T. F. Havel, N. Boulant, G. Boutis, E. Fortunato, S. Lloyd, R. Martinez, C. Negrevergne, M. Pravia, Y. Sharf, G. Teklemariam, Y. S. Weinstein, and W. H. Zurek, Fortschr. Phys. 48, 875 (2000).

${ }^{57}$ A. Goto, R. Miyabe, K. Hashi, T. Shimizu, S. Ohki, G. Kido, and S. Machida, Jpn. J. Appl. Phys., Part 1 42, 2864 (2003).

${ }^{58}$ M. S. Anwar, D. Blazina, H. Carteret, S. B. Duckett, T. K. Halstead, J. A. Jones, C. M. Kozak, and R. J. K. Taylor, Phys. Rev. Lett. 93, 040501 (2004).

${ }^{59}$ H. J. Cho, J. Baugh, C. A. Ryan, D. G. Cory, and C. Ramanathan, J. Magn. Reson. 187, 242 (2007).

${ }^{60}$ S. L. Braunstein, C. M. Caves, R. Jozsa, N. Linden, S. Popescu, and R. Schack, Phys. Rev. Lett. 83, 1054 (1999).

${ }^{61}$ R. Rahimi, K. Takeda, M. Ozawa, and M. Kitagawa, J. Phys. A 39, 2151 (2006).

${ }^{62}$ A. Kagawa, Y. Murokawa, K. Takeda, and M. Kitagawa, J. Magn. Reson. 197, 9 (2009).

${ }^{63}$ C. Song, K.-N. Hu, C.-G. Joo, T. M. Swager, and R. G. Griffin, J. Am. Chem. Soc. 128, 11385 (2006). 